\title{
NBursts+phot: parametric recovery of galaxy star formation histories from the simultaneous fitting of spectra and broad-band spectral energy distributions
}

\author{
Igor V. Chilingarian† and Ivan Yu. Katkov \\ Sternberg Astronomical Institute, Moscow State University, \\ Universitetskii pr. 13, Moscow, 119992 Russia \\ email IC: chil@sai.msu.ru; IK: katkov.ivan@gmail.com
}

\begin{abstract}
We present NBURSTS+ PHOT, a novel technique for the parametric inversion of spectrophotometric data for unresolved stellar populations where high-resolution spectra and broadband SEDs are fitted simultaneously, helping to break the degeneracies between parameters of multi-component stellar population models.
\end{abstract}

Keywords. Methods: data analysis, galaxies: kinematics and dynamics, galaxies: stellar content.

\section{Motivation}

Optical spectra of galaxies contain important information about their internal kinematics and stellar content that can be extracted by fitting them using stellar population models. There are a number of approaches for fitting the full spectrum that have proved to be efficient in studying different classes of galaxies and star clusters.

On the other hand, the broad-band photometry in different spectral domains that became available for large samples of galaxies thanks to modern wide-field surveys allows one to study certain properties that cannot be derived from the spectra (e.g. internal extinction) and to break degeneracies between parameters in the case of complex star formation histories (SFH). Usually, photometric measurements and spectra are used independently. Here we propose a new approach NBuRsTS+PHOT that fits in a single minimization loop spectral and photometric information, recovering both the parametric $\mathrm{SFH}$, and internal kinematics of galaxies.

\section{Novel Technique}

NBuRsts+PHOT is the extension of the NBuRsts (Chilingarian et al. 2007a,b) parametric full spectrum fitting technique implemented quite similarly to the non-parametric spectrophotometric inversion proposed by Pappalardo et al. (2010). During the minimization, the $\chi^{2}$ value is computed as a sum of the spectral and photometric contributions taken with a certain weight $\alpha$ :

$$
\begin{array}{r}
\chi^{2}=\sum_{N_{\lambda}} \frac{\left(F_{i}-P_{1 p}\left(T_{i}(S F H) \otimes \mathcal{L}\left(v, \sigma, h_{3}, h_{4}\right)+P_{2 q}\right)\right)^{2}}{\Delta F_{i}^{2}}+\alpha \sum_{N_{p h}} \frac{\left(f_{j}-w_{j} t_{j}(S F H) A_{j}\right)^{2}}{\Delta f_{j}^{2}}, \\
\text { where } T_{i}(S F H)=\sum_{N_{\text {bursts }}} k_{i} T_{i}\left(t_{n}, Z_{n}\right)
\end{array}
$$

$\dagger$ Present address: CfA/SAO 60 Garden St., Cambridge, MA, 02138 USA 

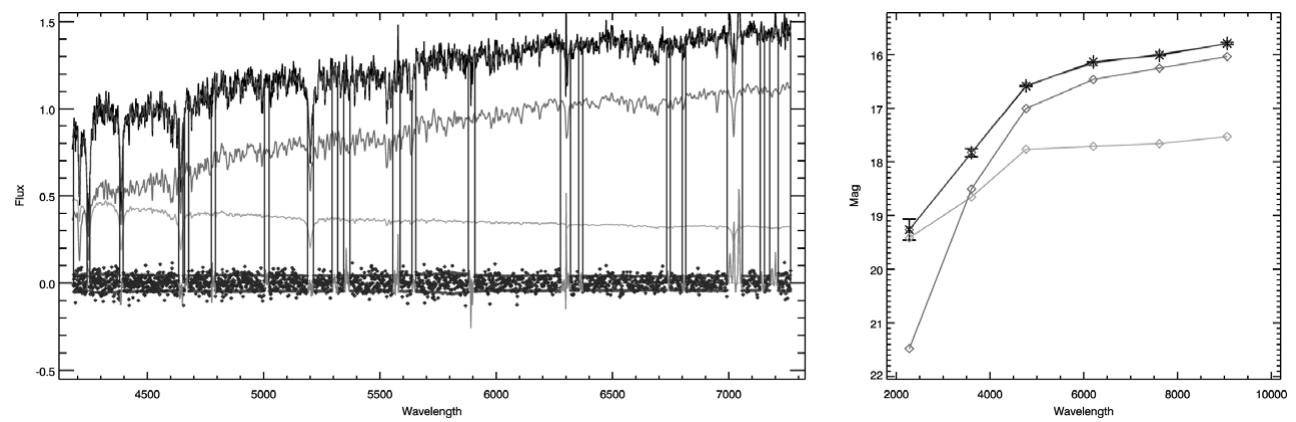

Figure 1. Example of the spectrophotometric inversion for a poststarburst E+A galaxy SDSS J230743.41+152558.4. The SDSS spectrum is shown in the left panel; the SED including GALEX NUV and 5 SDSS photometric measurements is shown in the right panel. Data (both spectroscopic and photometric) and the best-fitting model are shown in black and dark-grey, respectively, and the two components of the model are shown in light-grey.

where $\mathcal{L}$ is the line-of-sight velocity dispersion, $F_{i}$ and $\Delta F_{i}$ are the observed spectral flux and its uncertainty, $T_{i}(S F H)$ is the flux from a synthetic spectrum, represented by a linear combination of $N_{\text {bursts }}$ SSPs and convolved according to the line-spread function of the spectrograph, $P_{1 p}$ and $P_{2 q}$ are multiplicative and additive Legendre polynomials of orders $p$ and $q$ for correcting the continuum, $t$ is the age, $Z$ the metallicity, and $v$, $\sigma, h_{3}$, and $h_{4}$ are the radial velocity, velocity dispersion and Gauss-Hermite coefficients respectively (van der Marel \& Franx, 1993). $f_{j}$ and $t_{j}$ are the observed and modelled photometric fluxes, $w_{j}$ is a photometric flux normalization coefficient computed linearly, and $A_{j}(E(B-V))$ is a photometric extinction in a given band depending on $E(B-V)$, an additional free parameter. By adjusting the $\alpha$ parameter, one can choose the relative importance of photometric and spectral measurements. When dealing with galaxies at non-zero redshifts, the photometric measurements should be $k$-corrected.

In Fig. 1 we present an application of the NBURSTS+PHOT technique to the combined dataset for a poststarburst E+A galaxy SDSS J230743.41+152558.4 (Chilingarian et al. 2009). We used its SDSS DR7 (Abazajian et al. 2009) spectrum (left panel) and photometry from SDSS DR7 (5 optical ugriz bands) and GALEX GR5 (one NUV band; Martin et al. 2005) surveys. Photometric measurements were $k$-corrected using the analytical approximations from Chilingarian et al. (2010) and Chilingarian \& Zolotukhin (2011) for optical and GALEX bands respectively†.

\section{Pros and Cons}

\subsection{Pros}

- Photometry and spectra are fitted in a single loop, so all the available information is used to constraint the SFH and internal kinematics. Therefore, certain biases originating from degeneracies between parameters of internal kinematics and stellar populations (such as metallicity - velocity dispersion) can be avoided.

- Photometry in certain bands helps to break degeneracies between parameters of multiple star formation episodes. For example, adding GALEX UV colours will help to detect small quantities of young stars and measure their mass fraction, while spectra will be helpful to recover their parameters (age and metallicity) which cannot be derived from photometric data alone.

$\dagger$ See the $k$-corrections calculator at http://kcor.sai.msu.ru/UVtoNIR.html 
- We can estimate the internal extinction in galaxies while constraining their stellar population properties by the spectral data.

\subsection{Cons}

- The choice of $\alpha$ is not evident. It has to be determined for every data collection.

- The spectral and photometric models have different origins and are constructed from different ingredients. Therefore, the procedure is not fully self-consistent and some biases can be introduced. For the spectral fitting we use high-resolution SSP models computed with the PEGASE.HR (Le Borgne et al. 2004) code based on the ELODIE.3.1 (Prugniel et al. 2007) empirical stellar library, while the photometric models are computed with the PEGASE.2 (Fioc \& Rocca-Volmerange 1997) code using the low-resolution BaSeL synthetic stellar library (Lejeune et al. 1997). Spectra of real galaxies often contain emission lines which are sometimes quite strong and may influence the corresponding photometric measurements.

- It is difficult to get homogeneous photometry in several spectral domains because the data provided by different wide-field surveys often have certain specific features different, such as apertures and photometric zero points. In addition, the photometry has to be provided for the same aperture as a spectrum (e.g. we cannot use the integrated galaxy photometry and 3-arcsec SDSS spectra).

The authors thank the IAU for the provided financial aid and RFBR grant 10-0200062a for covering the remaining expanses.

\section{References}

Abazajian, K. N., et al. 2009, ApJS, 182, 543

Chilingarian, I. V., De Rijcke, S., \& Buyle, P. 2009, ApJL, 697, L111

Chilingarian, I. V., Melchior, A.-L., \& Zolotukhin, I. Y. 2010, MNRAS, 405, 1409

Chilingarian, I. V., Prugniel, P., Sil'chenko, O. K., \& Afanasiev, V. 2007, MNRAS, 376, 1033

Chilingarian, I. V., Prugniel, P., Sil'chenko, O. K., \& Koleva, M. 2007, in: Vazdekis, A. \& Peletier, R.R., eds., Proc. IAU Symp. 241, "Stellar Populations as Building Blocks of Galaxies". Cambridge Univ. Press, Cambridge, p. 175

Chilingarian, I. V. \& Zolotukhin, I. Y. 2011, MNRAS (in press)

Fioc, M. \& Rocca-Volmerange, B. 1997, A\&A, 326, 950

Le Borgne, D., Rocca-Volmerange, B., Prugniel, P., Lançon, A., Fioc, M., \& Soubiran, C. 2004, $A \xi A, 425,881$

Lejeune, T., Cuisinier, F., \& Buser, R. 1997, A\&SAS, 125, 229

Martin, D. C., et al. 2005, ApJL, 619, L1

Pappalardo, C., Lançon, A., Vollmer, B., Ocvirk, P., Boissier, S., \& Boselli, A. 2010, A\&\&A, 514, A33

Prugniel, P., Soubiran, C., Koleva, M., \& Le Borgne, D. 2007, astro-ph/0703658

van der Marel, R. \& Franx, M. 1993, ApJ, 407, 525 